\title{
Intracerebral Hemorrhage Recurrence in Patients with and without Cerebral Amyloid Angiopathy
}

\author{
João Pinho $^{a}$ José Manuel Araújo ${ }^{b}$ Ana Sofia Costa ${ }^{a, c}$ Fátima Silva $^{d, e}$ \\ Alexandra Francisco $^{d, e}$ Miguel Quintas-Neves ${ }^{f}$ João Soares-Fernandes ${ }^{f}$ \\ Carla Ferreirab $^{b}$ Tiago Gil Oliveira ${ }^{e, f}$ \\ ${ }^{a}$ Department of Neurology, University Hospital RWTH Aachen, Aachen, Germany; ${ }^{b}$ Department of Neurology, \\ Hospital de Braga, Braga, Portugal; ' JARA Institute Molecular Neuroscience and Neuroimaging, Forschungszentrum \\ Jülich and RWTH Aachen University, Aachen, Germany; ${ }^{\mathrm{d}}$ Department of Informatics, University of Minho, Braga, \\ Portugal; ' Life and Health Sciences Research Institute (ICVS), ICVS/3B's, School of Medicine, University of Minho,

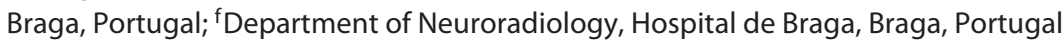

\author{
Keywords \\ Intracerebral hemorrhage - Recurrence $\cdot$ Cerebral amyloid \\ angiopathy
}

\begin{abstract}
Background: Intracerebral hemorrhage (ICH) recurrence risk is known to be higher in patients with cerebral amyloid angiopathy (CAA) as compared to other causes of ICH. Risk factors for ICH recurrence are not completely understood, and our goal was to study specific imaging microangiopathy markers. Methods: Retrospective case-control study of patients with non-traumatic $\mathrm{ICH}$ admitted to a single center between 2014 and 2017 who underwent magnetic resonance imaging (MRI). Clinical characteristics of the index event and occurrence of death and $\mathrm{ICH}$ recurrence were collected from clinical records. MRI images were independently reviewed by 2 neuroradiologists. Groups of patients with CAA-related and CAA-unrelated ICH defined were compared. Presence of CAA was defined according to the Boston modified criteria. Survival analysis with Kaplan-Meier curves and Cox-regression analyses was performed to analyze ICH
\end{abstract}

recurrence-free survival. Results: Among 448 consecutive patients with non-traumatic $\mathrm{ICH}$ admitted during the study period, 104 were included in the study, mean age 64 years $( \pm 13.5)$, median follow-up of 27 months (interquartile range, IQR 16-43), corresponding to 272 person-years of total follow-up. CAA-related ICH patients presented higher burden of lobar microbleeds $(p<0.001)$, higher burden of enlarged perivascular spaces (EPVS) in centrum semiovale $(p<0.001)$ and more frequently presented cortical superficial siderosis (cSS; $p<0.001$ ). ICH recurrence in patients with CAA was 12.7 per 100 person-years, and no recurrence was observed in patients without CAA. Variables associated with $\mathrm{ICH}$ recurrence in the whole population were age (hazard ratio [HR] per 1-year increment $=1.05,95 \% \mathrm{Cl} 1.00-1.11, p=0.046$ ), presence of disseminated cSS (HR 3.32, 95\% Cl 1.09-10.15, $p=0.035$ ) and burden of EPVS in the centrum semiovale (HR per 1-point increment $=1.80,95 \% \mathrm{Cl} 1.04-3.12, p=0.035$ ). Conclusions: This study confirms a higher ICH recurrence risk in patients with CAA-related ICH and suggests that age, disseminated CSS, and burden of EPVS in the centrum semiovale are associated with $\mathrm{ICH}$ recurrence.

C 2021 The Author(s)

Published by S. Karger AG, Basel 


\section{Introduction}

Arteriolosclerotic microangiopathy and cerebral amyloid angiopathy (CAA) are among the most frequent causes of non-traumatic intracerebral hemorrhage (ICH) [1]. It is well known that CAA is associated with a high hemorrhage recurrence risk (up to $10 \%$ per year) [2], and that this risk is significantly higher when compared to patients with ICH related to other causes $[3,4]$. The distribution of cerebral microbleeds is thought to reflect the etiology of the underlying microangiopathy, which translates into different hemorrhage recurrence risks, but the role of other in vivo biomarkers of recurrence has also been highlighted in more recent studies $[5,6]$. Characterization of new imaging biomarkers of ICH recurrence may not only provide better prognostic information in patients with ICH at index event but may also help us to better understand the pathophysiology of the underlying cause. Our goal was to study ICH recurrence in patients with CAA-related and $\mathrm{CAA}$-unrelated $\mathrm{ICH}$, and to analyze factors associated with $\mathrm{ICH}$ recurrence irrespective of etiology, specifically magnetic resonance imaging (MRI) markers such as burden of enlarged perivascular spaces (EPVS) and white matter hyperintensities.

\section{Methods}

This was a retrospective case-control study of adult patients with non-traumatic ICH admitted to a single university hospital between 2014 and 2017. Records were retrieved according to ICD-
9 and ICD-10 primary or secondary diagnosis of ICH. Patients with other diagnoses and underlying structural lesions such as vascular malformations and cerebral tumors were excluded after review of individual patient records. All patients who underwent brain MRI (including T1-, T2-, and T2*-weighted images and T2FLAIR) were included in the study. All patients underwent 1.5-tesla MRI, and the MRI acquisition parameters were constant throughout the study period. Patients who did not undergo all of the aforementioned sequences, whose images were not available or whose images were of low quality for analysis were excluded. Demographic and clinical variables for the index event, and occurrence of death and non-traumatic ICH recurrence were collected from the digital clinical records. Patients were followed in our Neurology outpatient clinic as part of the routine clinical care, and information concerning death and $\mathrm{ICH}$ recurrence was collected from the digital clinical records (registration of death, subsequent in-hospital admission records, outpatient clinic records).

MRI images were independently reviewed by 2 senior neuroradiologists for the classification of the following: microbleed anatomical rating scale [7]; presence of cortical superficial siderosis $(\mathrm{cSS}$; focal $=$ restricted to 3 sulci, disseminated $=$ involving more than 3 sulci) [8]; EPVS score (score $0=$ no EPVS, score $1=<10$ EPVS, score 2 = 11-20 EPVS, score 3=21-40 EPVS, score $4=>40$ EPVS) [9]; and age-related white matter changes (ARWMC; rating for periventricular white matter changes: score $0=$ no lesions, score 1 = focal lesions, score 2 = beginning confluence of lesions, score 3 = diffuse involvement of the entire region; rating for deep white matter changes: score $0=$ no lesions, score $1=1$ focal lesion $(\geq 5$ $\mathrm{mm}$ ), score $2=>1$ focal lesion, score 3 = confluent lesions) [10]. Interobserver agreement was calculated using interclass correlation coefficient (ICC) for single measures with 95\% CI. Disagreements were reviewed by the 2 raters and a consensus was reached. Patients were classified as CAA-related ICH if criteria for possible or probable CAA according to the modified Boston criteria [8] were fulfilled. Within the group with CAA-unrelated $\mathrm{ICH}$, patients with mixed-location hemorrhage/microbleeds were identified [11].

Fig. 1. Patient selection flowchart.

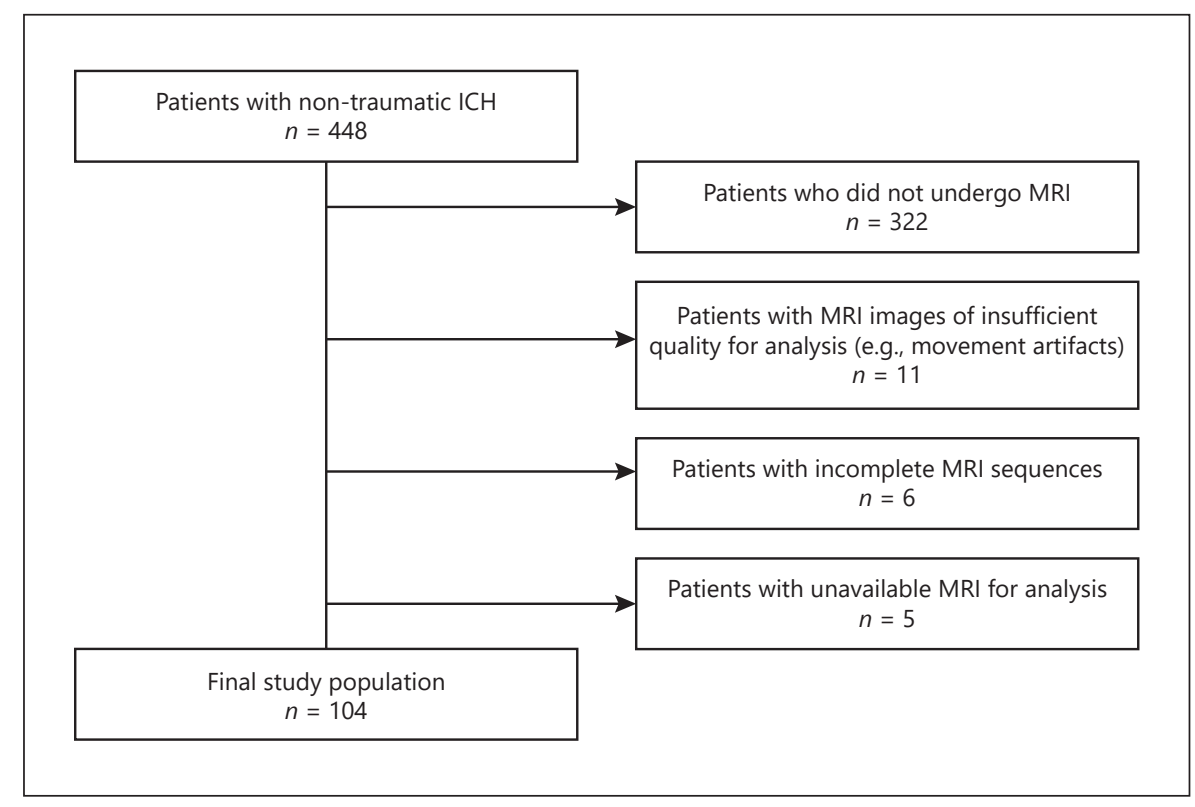


Baseline variables of the patients with CAA-related $\mathrm{ICH}$ and CAA-unrelated ICH were compared using $\chi^{2}$ test, independent sample $t$ test and Mann-Whitney test as appropriate. Kaplan-Meier curves for recurrence of ICH were constructed and Mantel-Cox test was used for group comparison. Univariable survival analyses for recurrence of $\mathrm{ICH}$ in the whole study population were performed using Cox regression. Variables flagged in the univariable analysis as significant were used to construct a multivariable Cox regression model. Survival time for Cox regression analyses was the time between hospital admission for index ICH and the occurrence of ICH recurrence, or loss to follow-up or death. Statistical significance was set as an alpha value of 0.05 . Anonymized data is available on request.

\section{Results}

Among 448 consecutive patients with non-traumatic ICH admitted during the study period, 126 patients underwent brain MRI. From these, 22 patients were excluded because of missing MRI sequences, insufficient quality, or unavailability of images for analysis (Fig. 1). Patients who were excluded from the study were older, more frequently had antiplatelet therapy at baseline, deep ICH and intraventricular rupture, and presented a significantly higher 30-day mortality (online suppl. Table 1, see

Table 1. Baseline characteristics of patients according to the presence of CAA-unrelated and CAA-related ICH

\begin{tabular}{|c|c|c|c|}
\hline & $\begin{array}{l}\text { CAA-unrelated } \\
\mathrm{ICH}(n=56)\end{array}$ & $\begin{array}{l}\text { CAA-related } \\
\mathrm{ICH}(n=48)\end{array}$ & $p$ value \\
\hline Age, years & $59.6 \pm 13.9$ & $70.9 \pm 10.2$ & 0.007 \\
\hline Male sex & $38(68.9)$ & $24(50.0)$ & 0.064 \\
\hline Hypertension & $40(71.4)$ & $36(75.0)$ & 0.825 \\
\hline Dyslipidemia & $27(48.2)$ & $28(58.3)$ & 0.303 \\
\hline Diabetes & $10(17.9)$ & $11(22.9)$ & 0.522 \\
\hline Antiplatelet therapy & $7(12.5)$ & $9(18.8)$ & 0.378 \\
\hline Anticoagulation therapy & $9(16.1)$ & $10(20.8)$ & 0.531 \\
\hline Admission Glasgow Coma Scale & $15(13-15)$ & $14(14-15)$ & 0.974 \\
\hline Admission systolic blood pressure, $\mathrm{mm} \mathrm{Hg}$ & $155(133-174)$ & $144(132-153)$ & 0.029 \\
\hline Admission diastolic blood pressure, $\mathrm{mm} \mathrm{Hg}$ & $87(69-101)$ & $75(67-83)$ & 0.006 \\
\hline Admission glucose, $\mathrm{mg} / \mathrm{dL}$ & $120(109-149)$ & $109(103-131)$ & 0.040 \\
\hline Admission INR & $1.0(1.0-1.2)$ & $1.1(1.0-1.2)$ & 0.880 \\
\hline Admission platelet count, $/ \mu \mathrm{L}$ & $211(153-254)$ & $201(167-230)$ & 0.719 \\
\hline \multicolumn{4}{|l|}{ ICH location } \\
\hline Lobar & $11(19.6)$ & $45(93.8)$ & $<0.001$ \\
\hline Deep & $33(58.9)$ & 0 & $<0.001$ \\
\hline Infratentorial & $12(21.4)$ & $3(6.3)$ & 0.047 \\
\hline Intraventricular rupture & $14(25.0)$ & $14(29.2)$ & 0.633 \\
\hline Emergent neurosurgery & $6(10.7)$ & $2(4.2)$ & 0.282 \\
\hline \multicolumn{4}{|l|}{ MRI findings } \\
\hline \multicolumn{4}{|l|}{ Age-related white matter changes score } \\
\hline Periventricular & $1(1-2)$ & $2(1-3)$ & 0.107 \\
\hline Deep & $1(1-2)$ & $2(1-3)$ & 0.142 \\
\hline \multicolumn{4}{|l|}{ Enlarged perivascular spaces score } \\
\hline Centrum semiovale & $1(1-2)$ & $2.5(1-3)$ & $<0.001$ \\
\hline Basal ganglia & $2(1-2)$ & $1(1-2)$ & 0.119 \\
\hline \multicolumn{4}{|l|}{ Number of microbleeds } \\
\hline Lobar & $0(0-1)$ & $5(1-9)$ & $<0.001$ \\
\hline Basal ganglia & $0(0-2)$ & - & $<0.001$ \\
\hline Infratentorial & $0(0-1)$ & $0(0-1)$ & 0.930 \\
\hline Cortical superficial siderosis & $4(7.1)$ & $28(58.3)$ & $<0.001$ \\
\hline Focal $(\leq 3$ sulci $)$ & $1(1.8)$ & $13(27.1)$ & $<0.001$ \\
\hline Disseminated (>3 sulci) & $3(5.4)$ & $15(31.3)$ & 0.001 \\
\hline 30-day mortality & $2(3.6)$ & $1(2.1)$ & 1.000 \\
\hline Follow-up, months & $27(21-43)$ & $24(14-43)$ & 0.396 \\
\hline ICH recurrence & 0 & $13(27.1)$ & $<0.001$ \\
\hline
\end{tabular}

Data is presented as $n(\%)$, mean $\pm \mathrm{SD}$, and median (interquartile range). CAA, cerebral amyloid angiopathy; $\mathrm{ICH}$, intracerebral hemorrhage; INR, international normalized ratio; MRI, magnetic resonance imaging. 


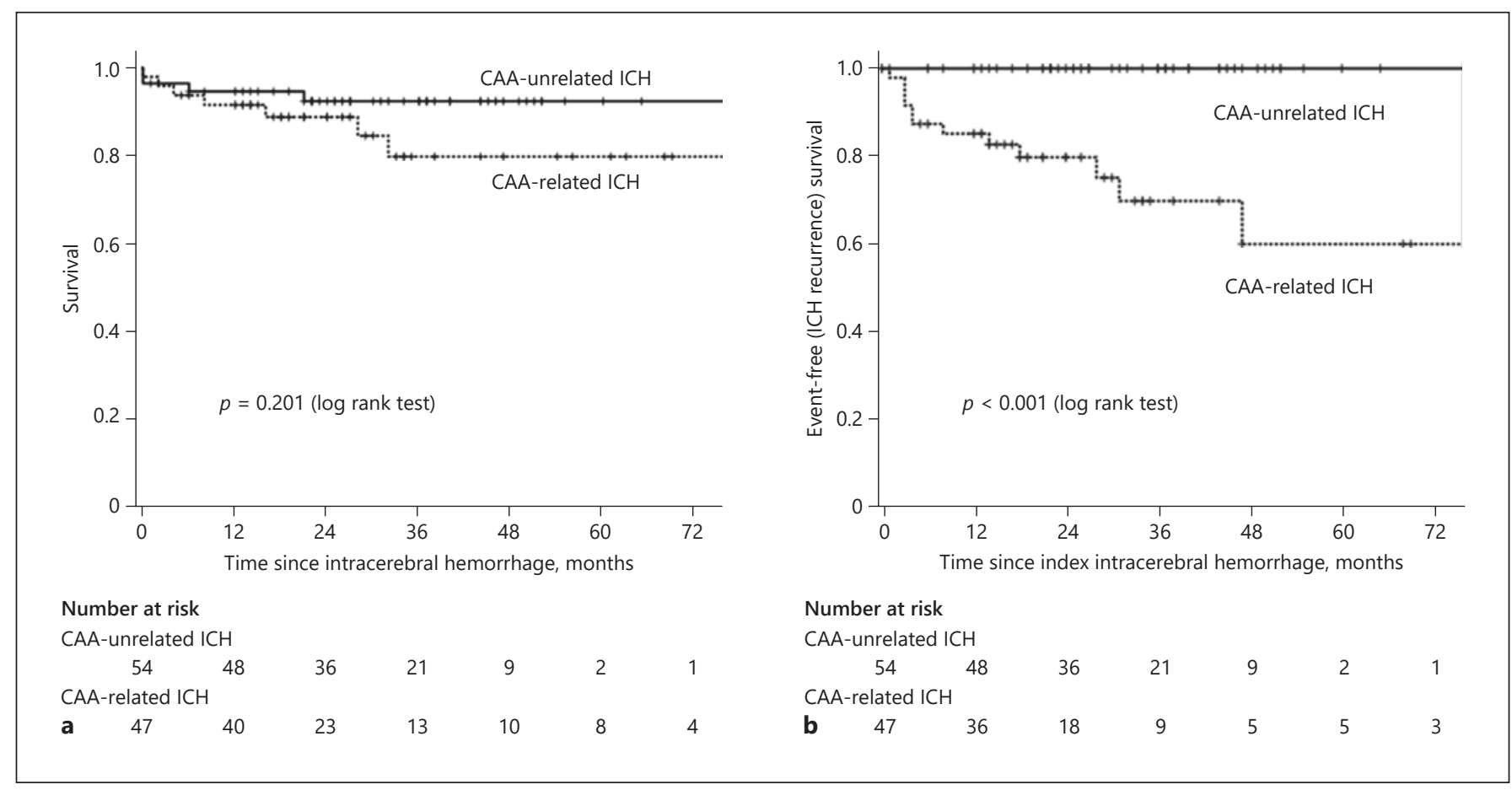

Fig. 2. Kaplan-Meier curves for survival (a) and for ICH recurrence (b) according to the presence of CAA-related and CAA-unrelated ICH.

www.karger.com/doi/10.1159/000513503). The final study population consisted of 104 patients, with a mean age of 64 years ( \pm 13.5$), 62(59.6 \%)$ of male sex, and a median follow-up time of 27 months (interquartile range 16-43) corresponding to 272 person-years. Overall ICH recurrence during follow-up was $12.5 \%$ (4.8 per 100 person-years). Interobserver agreement for MRI variables was good to excellent (lowest ICC $=0.84$ for deep ARWMC; higher ICC $=0.99$ for counting of lobar microbleeds).

Baseline characteristics of the groups with CAA-unrelated ICH $(n=56)$ and CAA-related ICH $(n=48)$ are presented in Table 1. Among patients with CAA-related $\mathrm{ICH}$, the majority had probable CAA $(n=41)$, and only 7 patients had possible CAA according to the modified Boston criteria. Patients with CAA-related ICH were older (mean age 70.9 vs. 59.6 years, $p=0.007$ ), presented lower systolic blood pressure (144 vs. $155 \mathrm{~mm} \mathrm{Hg}, p=$ 0.029 ), lower diastolic blood pressure ( 75 vs. $87 \mathrm{~mm} \mathrm{Hg}$, $p=0.006$ ), and lower blood glucose (109 vs. $202 \mathrm{mg} / \mathrm{dL}$, $p=0.040)$ on admission. As expected, patients with CAArelated ICH more frequently presented lobar hemorrhages at the index event ( 93.8 vs. $19.6 \%, p<0.001)$. Three patients with CAA-related ICH presented a cerebellar hemorrhage which was, in the 3 patients, superficial according to Pasi et al. [12]. Concerning MRI findings, patients with CAA-related $\mathrm{ICH}$ presented higher burden of lobar microbleeds (median number of lobar microbleeds 5 vs. $0, p<0.001$ ), higher burden of EPVS in centrum semiovale (median EPVS score 2.5 vs. $1, p<0.001$ ) and more frequently presented cSS (58.3 vs. $7.1 \%, p<0.001)$. Among patients with CAA-unrelated $\mathrm{ICH}$, we found 25 patients $(44.6 \%)$ with mixed-location hemorrhage/microbleeds.

None of the patients with CAA-unrelated ICH experienced hemorrhage recurrence, while 13 patients with CAA-related ICH had at least a hemorrhage recurrence during the follow-up period (27.1\%, 12.7 per 100 personyears). The Kaplan-Meier curves showed no difference concerning mortality between groups $(p=0.201)$ but showed significantly higher ICH recurrence in the group of patients with CAA-related ICH ( $p<0.001$; Fig. 2).

In the univariable survival analyses (Table 2), the only variables associated with ICH recurrence were age (hazard ratio $[\mathrm{HR}]$ per 1 -year increment $=1.05,95 \%$ CI 1.00 $1.11, p=0.046)$, presence of disseminated cSS (HR 3.32, $95 \%$ CI 1.09-10.15, $p=0.035$ ), and burden of EPVS in the centrum semiovale (HR per 1 -point increment $=1.80$,
18

Cerebrovasc Dis Extra 2021;11:15-21 DOI: $10.1159 / 000513503$
Pinho et al. 
Table 2. Univariable and multivariable survival analyses using ICH recurrence during follow-up as endpoint in the Cox regression

\begin{tabular}{|c|c|c|c|c|}
\hline & $\begin{array}{l}\text { Univariable } \\
\text { hazard ratio }(95 \% \mathrm{CI})\end{array}$ & $p$ value & $\begin{array}{l}\text { Multivariable } \\
\text { hazard ratio }(95 \% \mathrm{CI})\end{array}$ & $p$ value \\
\hline Age (per 1 year increase) & $1.05(1.00-1.11)$ & 0.046 & $1.03(0.97-1.09)$ & 0.318 \\
\hline Male sex & $0.82(0.28-2.45)$ & 0.722 & & \\
\hline Hypertension & $0.69(0.21-2.30)$ & 0.548 & & \\
\hline Baseline systolic BP (per $10 \mathrm{~mm} \mathrm{Hg}$ increment) & $0.92(0.71-1.19)$ & 0.533 & & \\
\hline Baseline diastolic BP (per $10 \mathrm{~mm} \mathrm{Hg}$ increment) & $0.82(0.56-1.22)$ & 0.331 & & \\
\hline Diabetes & $1.48(0.45-4.91)$ & 0.523 & & \\
\hline Admission glucose (per $10 \mathrm{mg} / \mathrm{dL}$ increment) & $0.79(0.62-1.01)$ & 0.057 & & \\
\hline Dyslipidemia & $1.21(0.40-3.62)$ & 0.736 & & \\
\hline Diabetes & $1.48(0.45-4.91)$ & 0.523 & & \\
\hline Baseline antiplatelet therapy & $1.04(0.22-4.86)$ & 0.958 & & \\
\hline Baseline anticoagulation & $0.46(0.06-3.60)$ & 0.461 & & \\
\hline Baseline ICH volume (per $1 \mathrm{~mL}$ increment) & $1.02(1.00-1.05)$ & 0.116 & & \\
\hline Deep MB (per 1 MB increase) & $0.97(0.88-1.07)$ & 0.511 & & \\
\hline Lobar MB (per 1 MB increase) & $0.98(0.94-1.03)$ & 0.472 & & \\
\hline$>5$ lobar $\mathrm{MB}$ & $1.64(0.49-5.44)$ & 0.422 & & \\
\hline Focal cSS ( $\leq 3$ sulci) & $1.67(0.44-6.40)$ & 0.451 & & \\
\hline Disseminated cSS ( $>3$ sulci) & $3.32(1.09-10.15)$ & 0.035 & $2.11(0.62-7.16)$ & 0.230 \\
\hline Periventricular ARWMC & $1.37(0.67-2.81)$ & 0.387 & & \\
\hline Deep ARWMC & $1.63(0.84-3.19)$ & 0.152 & & \\
\hline EPVS centrum semiovale & $1.80(1.04-3.12)$ & 0.035 & $1.42(0.78-2.61)$ & 0.254 \\
\hline EPVS basal ganglia & $0.53(0.20-1.37)$ & 0.189 & & \\
\hline
\end{tabular}

95\% CI, 95\% confidence interval; BP, blood pressure; ICH, intracerebral hemorrhage; MB, microbleed; cSS, cortical superficial siderosis; ARWMC, age-related white matter hyperintensities; EPVS, enlarged perivascular spaces.

95\% CI 1.04-3.12, $p=0.035)$. In the multivariable regression model, no variable was independently associated with ICH recurrence (Table 2).

\section{Discussion}

This study confirms that ICH recurrence in patients with CAA-related ICH is significantly higher than in patients with CAA-unrelated ICH and found that increasing age, presence of disseminated cSS, and higher burden of EPVS in the centrum semiovale were associated with $\mathrm{ICH}$ recurrence in the whole study population.

The incidence of $\mathrm{ICH}$ recurrence in patients with CAA-related ICH is similar to larger cohort studies which reported incidences between 8 and 10\% per year [13-15]. However, we did not find any ICH recurrence in the group with CAA-unrelated ICH. This may be explained by the small group size, considering that, according to the incidence of ICH recurrence reported in a recent metaanalysis [3], we would expect to find only 1 patient with recurrence in our group of CAA-unrelated ICH.
Even though an increasing burden of microbleeds has been associated with ICH recurrence both in CAA-related and CAA-unrelated ICH [3], more recent cohorts of CAA patients did not find this association and highlighted the importance of cSS as a more robust predictor for ICH recurrence $[5,6,15]$. In a meta-analysis of studies which included patients with CAA with and without $\mathrm{ICH}$, even though cSS was found to be associated with occurrence of future ICH, disseminated cSS conferred a higher independent future ICH risk when compared to focal cSS [16]. Our results concerning the association of disseminated cSS with ICH recurrence are in line with these recent findings.

Few studies have addressed the role of other MRI microangiopathy markers as predictors of ICH recurrence [17]. The finding that a higher burden of EPVS in centrum semiovale is associated with ICH recurrence was only found in another larger study and may be a clue to better understanding the physiopathology of ICH in CAA. In a large single center cohort of CAA-related ICH survivors, Boulouis et al. [6] found that the total burden of microangiopathy (measured by the CAA-SVD-Score) was associated with a higher ICH recurrence, and that 
this association was mainly driven by the presence of cSS and higher burden of EPVS in the centrum semiovale. Perivascular spaces have been speculated to be involved in the pathogenesis of CAA, namely through dysfunction of amyloid- $\beta$ clearance pathways [18]. It has been found that a higher burden of juxtacortical EPVS in patients with CAA was associated with increased severity of cortical accumulation of amyloid- $\beta$ in vessel walls in the same regions [19], which could explain the association we found between EPVS in centrum semiovale and ICH recurrence risk. However, 2 other studies did not find such association between the severity of EPVS and recurrence risk in CAA-related ICH patients $[14,20]$, which warrants larger multicentric cohorts and meta-analyses to clarify this potential association.

The prevalence of hypertension and diabetes was similar in both groups of patients, which is probably related to the fact that the group with CAA-related ICH was significantly older, and raises the question of a possible contribution of arteriosclerotic microangiopathic changes for ICH also in this group of patients. However, it is interesting to note that systolic and diastolic blood pressures and glucose at admission were higher in patients with CAA-unrelated ICH, which suggests their contribution to the acute event, rather than a mere physiological reaction after ICH [21]. Inadequate blood pressure control after the acute phase of ICH is also known to be associated with $\mathrm{ICH}$ recurrence not only in patients with deep ICH, but also in patients with lobar ICH [22]. Unfortunately, we could not explore the contribution of blood pressure control to ICH recurrence because no systematic evaluation of blood pressure was carried out during follow-up in the routine clinical practice.

The main limitations of our study are related to its retrospective nature, its small sample size, possible recurrence detection bias in patients who die outside the hospital or who are admitted for ICH in other hospitals, low number of ICH recurrences, absence of ICH recurrence in the group of CAA-unrelated $\mathrm{ICH}$, and exclusion of ICH patients who did not undergo MRI, which is reflected in the low mortality of included patients and represents a selection bias. We did not analyze recurrence predictors only in the subgroup of patients with CAA-related ICH because of the small sample size, and this could represent a methodological limitation because recurrences were only observed in this group. Our results concerning ICH recurrence predictors are derived from our whole study population of ICH patients irrespective of etiology, and even though none of the analyzed clinical or imaging marker is specific for CAA, and is also seen in patients with other ICH etiologies, our results must be interpreted with caution. The strengths of the study lie in the complete baseline clinical characterization, relatively long mean follow-up period in the Neurology outpatient clinic, as well as good interobserver agreement for MRI microangiopathy markers.

In conclusion, ICH recurrence is more frequent in patients with CAA-related ICH when compared with patients with CAA-unrelated $\mathrm{ICH}$, and the most important predictors for ICH recurrence are the presence of cSS and higher burden of EPVS in the centrum semiovale.

\section{Statement of Ethics}

The study was approved by the Ethics for Health Commission of Hospital de Braga.

\section{Conflict of Interest Statement}

Authors report no disclosures.

\section{Funding Sources}

There are no funding sources to declare.

\section{Author Contributions}

J.P.: first manuscript draft writing; study design; data collection; statistical analysis. J.M.A., M.Q.-N., and J.S.-F.: final manuscript review; data collection. A.S.C.: final manuscript review; data collection; statistical analysis. F.S. and A.F.: final manuscript review; data processing and analysis. C.F.: final manuscript review; study design; important intellectual contribution. T.G.O.: final manuscript review; data collection; important intellectual contribution.

References

1 Pantoni L. Cerebral small vessel disease: from pathogenesis and clinical characteristics to therapeutic challenges. Lancet Neurol. 2010 Jul;9(7):689-701.

2 Charidimou A, Boulouis G, Gurol ME, Ayata C, Bacskai BJ, Frosch MP, et al. Emerging concepts in sporadic cerebral amyloid angiopathy. Brain. 2017 Jul;140(7):1829-50.

3 Charidimou A, Imaizumi T, Moulin S, Biffi A, Samarasekera N, Yakushiji Y, et al. Brain hemorrhage recurrence, small vessel disease type, and cerebral microbleeds: A meta-analysis. Neurology. 2017 Aug;89(8):820-9.
Cerebrovasc Dis Extra 2021;11:15-21 DOI: $10.1159 / 000513503$
Pinho et al. 
4 Pinho J, Costa AS, Araújo JM, Amorim JM, Ferreira C. Intracerebral hemorrhage outcome: A comprehensive update. J Neurol Sci. 2019 Mar;398:54-66.

5 Charidimou A, Boulouis G, Roongpiboonsopit D, Xiong L, Pasi M, Schwab KM, et al. Cortical superficial siderosis and recurrent intracerebral hemorrhage risk in cerebral amyloid angiopathy: large prospective cohort and preliminary meta-analysis. Int J Stroke. 2019 Oct; 14(7):723-33.

6 Boulouis G, Charidimou A, Pasi M, Roongpiboonsopit D, Xiong L, Auriel E, et al. Hemorrhage recurrence risk factors in cerebral amyloid angiopathy: comparative analysis of the overall small vessel disease severity score versus individual neuroimaging markers. J Neurol Sci. 2017 Sep;380:64-7.

7 Gregoire SM, Chaudhary UJ, Brown MM, Yousry TA, Kallis C, Jäger HR, et al. The Microbleed Anatomical Rating Scale (MARS): reliability of a tool to map brain microbleeds. Neurology. 2009 Nov;73(21):1759-66.

8 Linn J, Halpin A, Demaerel P, Ruhland J, Giese AD, Dichgans M, et al. Prevalence of superficial siderosis in patients with cerebral amyloid angiopathy. Neurology. 2010 Apr; 74(17):1346-50.

9 Doubal FN, MacLullich AM, Ferguson KJ, Dennis MS, Wardlaw JM. Enlarged perivascular spaces on MRI are a feature of cerebral small vessel disease. Stroke. 2010 Mar;41(3): $450-4$.
10 Wahlund LO, Barkhof F, Fazekas F, Bronge L, Augustin M, Sjögren M, et al.; European Task Force on Age-Related White Matter Changes. A new rating scale for age-related white matter changes applicable to MRI and CT. Stroke. 2001 Jun;32(6):1318-22.

11 Pasi M, Charidimou A, Boulouis G, Auriel E, Ayres A, Schwab KM, et al. Mixed-location cerebral hemorrhage/microbleeds: underlying microangiopathy and recurrence risk Neurology. 2018 Jan;90(2):e119-26.

12 Pasi M, Marini S, Morotti A, Boulouis G, Xiong L, Charidimou A, et al. Cerebellar hematoma location: implications for the underlying microangiopathy. Stroke. 2018 Jan; 49(1):207-10

13 Biffi A, Halpin A, Towfighi A, Gilson A, Busl $\mathrm{K}$, Rost N, et al. Aspirin and recurrent intracerebral hemorrhage in cerebral amyloid angiopathy. Neurology. 2010 Aug;75(8):693-8.

14 Wollenweber FA, Opherk C, Zedde M, Catak C, Malik R, Duering M, et al. Prognostic relevance of cortical superficial siderosis in cerebral amyloid angiopathy. Neurology. 2019 Feb;92(8):e792-801.

15 Raposo N, Charidimou A, Roongpiboonsopit D, Onyekaba M, Gurol ME, Rosand J, et al. Convexity subarachnoid hemorrhage in lobar intracerebral hemorrhage: A prognostic marker. Neurology 2020;94:e968-77.

16 Charidimou A, Boulouis G, Greenberg SM, Viswanathan A. Cortical superficial siderosis and bleeding risk in cerebral amyloid angiopathy: A meta-analysis. Neurology. 2019 Dec; 93(24):e2192-202.
17 Charidimou A, Boulouis G. Intracerebral haemorrhage recurrence in cerebral amyloid angiopathy: time to look beyond microbleeds? J Neurol Sci. 2016 Aug;367:213-4.

18 Wardlaw JM, Benveniste H, Nedergaard M, Zlokovic BV, Mestre $\mathrm{H}$, Lee $\mathrm{H}$, et al.; colleagues from the Fondation Leducq Transatlantic Network of Excellence on the Role of the Perivascular Space in Cerebral Small Vessel Disease. Perivascular spaces in the brain: anatomy, physiology and pathology. Nat Rev Neurol. 2020 Mar;16(3):137-53.

19 van Veluw SJ, Biessels GJ, Bouvy WH, Spliet WG, Zwanenburg JJ, Luijten PR, et al. Cerebral amyloid angiopathy severity is linked to dilation of juxtacortical perivascular spaces. J Cereb Blood Flow Metab. 2016 Mar;36(3): $576-80$

20 Koo HW, Jo KI, Yeon JY, Kim JS, Hong SC Clinical features of high-degree centrum semiovale-perivascular spaces in cerebral amyloid angiopathy. J Neurol Sci. 2016 Aug;367: 89-94.

21 Lin J, Piran P, Lerario MP, Ong H, Gupta A, Murthy SB, et al. Differences in admission blood pressure among causes of intracerebral hemorrhage. Stroke. 2020 Feb;51(2):644-7.

22 Biffi A, Anderson CD, Battey TW, Ayres AM, Greenberg SM, Viswanathan A, et al. Association between blood pressure control and risk of recurrent intracerebral hemorrhage. JAMA. 2015 Sep;314(9):904-12. 